Check for updates

Cite this: RSC Adv., 2017, 7, 31610

DOI: $10.1039 / c 7 r a 90070 h$

www.rsc.org/advances

\section{Correction: Spectroscopic studies on the comparative refolding of guanidinium hydrochloride denatured hen egg-white lysozyme and Rhizopus niveus lipase assisted by cationic single-chain/gemini surfactants via artificial chaperone protocol}

\author{
Nuzhat Gull, ${ }^{* a}$ Mohd Ishtikhar, ${ }^{\text {bd }}$ Md. Sayem Alam, ${ }^{c}$ Syedah Noorul Sabah Andrabi ${ }^{a}$ \\ and Rizwan Hasan Khan*d \\ Correction for 'Spectroscopic studies on the comparative refolding of guanidinium hydrochloride \\ denatured hen egg-white lysozyme and Rhizopus niveus lipase assisted by cationic single-chain/gemini \\ surfactants via artificial chaperone protocol' by Nuzhat Gull et al., RSC Adv., 2017, 7, 28452-28460.
}

The authors regret that the original Acknowledgements section did not acknowledge the Indian Institute of Technology Bombay for an Institute Post-Doctoral Fellowship. The corrected acknowledgements has been included below.

The Royal Society of Chemistry apologises for these errors and any consequent inconvenience to authors and readers.

\title{
Acknowledgements
}

Financial assistance from the Indian Council of Medical Research (ICMR) Government of India, ICMR-SRF project No. BIC/11/12/ 2013, in the form of a research fellowship and Dept. of Chemistry, Indian Institute of Technology Bombay for the fellowship in the form of Institute Post-Doctoral Fellowship to M. I. are the gratefully acknowledged. Encouragement from the Principal and faculty Dept. of Chemistry, Govt. Degree College for Women, M. A. Road, Srinagar to N. G. and S. N. S. is gratefully acknowledged. We are thankful to Interdisciplinary Biotechnology Unit, Aligarh Muslim University, Aligarh for the use of their facilities.

\footnotetext{
${ }^{a}$ Department of Chemistry, Govt. Degree College for Women, M. A. Road, Srinagar-190001, India. E-mail: nuzhatwahid@gmail.com; Tel: +91-9697508005

${ }^{b}$ Department of Chemistry, Indian Institute of Technology Bombay, Powai, Mumbai, India

${ }^{c}$ Central Leather Research Institute, Chennai, India

${ }^{d}$ Interdisciplinary Biotechnology Unit, Aligarh Muslim University, Aligarh-202002, India. E-mail: rizwanhkhan1@gmail.com; Fax: +91-571-2721776; Tel: +91-571-2727388
} 